\title{
Clase Magistral del Embajador de Brasil, Excmo. Sr. Georges Lamazière
}

Realizada el 24 de noviembre de 2014, con motivo de la Cremonia de Egreso de la XXIV promoción del Magíster en Estudios Internacionales (MEI) y la III promoción del Magíster en Estrategia Internacional y Política Comercial (MEIPC).

Desde la redemocratización, Brasil mantiene una línea de acción en política externa que se puede decir constante, con variaciones de matices, pero no de estructura o de sentido. Estas líneas generales obedecen a la propia Constitución Federal de 1988, que en su artículo $4^{\circ}$ dispone sobre los principios que el Poder Ejecutivo debe observar en la conducción de las relaciones internacionales del país. Podemos citar entre nuestras prioridades la integración regional, la valorización de la gobernanza multilateral, tanto política como económicocomercial, en particular del sistema ONU, la profundización de las relaciones con el mundo en desarrollo y la prevalencia de los derechos humanos. Otros temas importantes que han sido objeto de atención calificada de la política externa brasileña son el calentamiento global, el combate al hambre y a la miseria, la promoción de los derechos de las mujeres y la nueva gobernanza global en el ámbito del G-20 y de los BRICS, entre otros.

Como dijo el ministro de Relaciones Exteriores de Brasil, Luiz Alberto Figueiredo, en su discurso de asunción al cargo: «El fortalecimiento de la presencia de Brasil en el exterior se viene realizando por medio de una acción diplomática que ha sabido conjugar el énfasis prioritario en la integración regional y en las relaciones con cada uno de los países vecinos, con una proyección de carácter global y verdaderamente universal, como es la vocación de nuestra política externa». 
La política externa brasileña se ha dedicado al perfeccionamiento de la gobernanza global por medio de la promoción de la reforma del sistema de las Naciones Unidas y de la creación de nuevas instancias que permitan a los países en desarrollo una mejor articulación y participación en el proceso decisorio. Son ejemplos concretos de esta última vertiente el establecimiento de nuevos foros como el IBAS (India, Brasil, África del Sur), los BRICS (Brasil, Rusia, India, China y África del Sur), ASPA (América del Sur-Países Árabes), y ASA (América del Sur-África). En la Organización Mundial del Comercio (OMC), Brasil ha liderado esfuerzos para la apertura de mercados, notoriamente en agrícola.

Desearía dedicar algunas palabras al tema de la reforma de las Naciones Unidas, y en particular de su Consejo de Seguridad. Como todos saben, este tema se encuentra en lo alto de la agenda de reforma, desde hace ya bastante tiempo. El mundo no puede prescindir de un Consejo de Seguridad que sea capaz de lidiar con las graves amenazas a la paz. El Consejo de Seguridad renovado debería reflejar la emergencia de nuevos actores, en particular del mundo en desarrollo, que sean capaces de contribuir a la superación de los desafíos de la agenda internacional.

Para citar nuevamente al canciller Figueiredo: «Cuando fue establecido el Consejo de Seguridad, en 1945, no solo el mundo era diferente, sino que países como la India ni siquiera eran independientes y estructurados. Alemania estaba destruida por la guerra, y Japón también. Estos países, hoy, son grandes actores internacionales, pero en aquella época no lo eran».

El congelamiento del Consejo de Seguridad en su formato establecido en 1945 ha resultado en importantes obstáculos a su funcionamiento y eficiencia, además de cuestionamientos acerca de su propia legitimidad. En aquella época eran solo 51 los miembros de las Naciones Unidas, mientras que hoy sumamos casi 200 países. Sería necesario decir que la gran mayoría de los nuevos miembros provienen del mundo en desarrollo y que su incorporación en las principales instancias decisivas no se ha dado con la rapidez esperada.

Brasil es candidato a un asiento permanente en el Consejo. Acogemos, con gran satisfacción, el apoyo de Chile, país con el cual Brasil ha mantenido un estrecho diálogo dentro de su 
ámbito, y de un gran número de naciones a la candidatura brasileña. A propósito, recuerdo que el canciller Heraldo Muñoz ofreció, de manera generosa, a nuestro país, la acreditación de un diplomático brasileño en la delegación chilena que ahora ocupa un asiento rotativo en el Consejo.

A cambio, Brasil, rápidamente ofreció a Chile transferir informaciones de su amplia red de puestos en el exterior, en particular en lo que refiere a países objeto de interés de las deliberaciones del Consejo y donde Chile no mantenga una legación.

En lo que concierne a América del Sur, nos dedicamos a la consolidación del proyecto de la Unión de Naciones Sudamericanas (UNASUR). Buscamos de esa forma la construcción de un espacio de articulación en el ámbito político de defensa, económico, social y cultural en nuestra región. También, destaco a propósito, el papel del Consejo Sudamericano de Infraestructura y Planeamiento (COSIPLAN), organismo accesorio de UNASUR, responsable de la coordinación de los esfuerzos de nuestros países con el objetivo de crear la infraestructura de la integración, en la cual los corredores bioceánicos son tan importantes para Brasil y para Chile. La presidenta Michelle Bachelet ha declarado que Chile pretende transformarse en "puerto y puente» entre la región del Asia Pacífico y la América del Sur atlántica. La COSIPLAN, actualmente bajo la presidencia pro tempore de Chile, constituye el foro de discusiones adecuado para alcanzar ese objetivo, entre otros.

Aquí es importante subrayar que cualesquiera sean los esquemas de integración económica escogidos por los países de América del Sur y sus vínculos comerciales y financieros con otras regiones del mundo, todas las demás dimensiones de la vida de sus pueblos serán determinadas, en gran medida, por el estado de la vecindad. Para comenzar por los temas de seguridad, sobre todo en las vertientes no tradicionales relacionadas con el narcotráfico, el terrorismo y muchas otras, donde la coordinación regional será crucial. Y continuando por la deseable estabilidad política, por el refuerzo de la democracia y el respeto a los derechos humanos, por el desarrollo sustentable, por el intercambio educacional, científico tecnológico, cultural, turístico, humano, en fin. Vivimos en el mismo barrio y tenemos que cuidar de él solidariamente. 
Una recordación dolorosa, pero convincente, de la evidencia de que compartimos un mismo espacio y un mismo tiempo, reside en el tema de los derechos humanos, que ha sido objeto de intensos intercambios entre nuestros dos países. Compartimos una historia común, una trayectoria marcada por la dictadura y por el proceso de redemocratización. Enfrentamos las mismas dificultades en la búsqueda de la reconciliación entre el pasado y el presente. Esa particularidad ha impulsado a nuestros dos gobiernos a intercambiar informaciones y documentos con el objetivo de conocer más detalladamente nuestra historia, así como dejar que surja la luz sobre las violaciones ocurridas durante el período dictatorial.

En ese sentido, en la semana del 21 al 25 de abril de 2014, una delegación brasileña de la Comisión Nacional de la Verdad estuvo en misión de trabajo en Chile, realizando investigaciones en archivos locales y reuniéndose con autoridades y personalidades civiles comprometidas con la lucha por la preservación de la memoria y defensa de los derechos humanos. Pocos meses después, en junio, fue firmado el Memorándum de Entendimiento para el Intercambio de Documentos para la Aclaración de Graves Violaciones a los Derechos Humanos, que facilitará la investigación en los dos países, permitiendo la profundización de las investigaciones sobre los crímenes ocurridos durante los regímenes dictatoriales.

Podemos afirmar, con seguridad, que la más relevante entre esas prioridades de acción externa continúa siendo la integración de América del Sur. Esa no es una tarea menor. Nuestro continente posee una historia de siglos de desconocimiento mutuo, cuando no de rivalidad y aún de conflictos. La construcción de un espacio de paz y armonía en nuestra región es obviamente de interés de todos los países sudamericanos, que solamente de esta manera podrán dedicarse plenamente a la tarea del desarrollo. Es, por tanto, de interés para nuestro país, que representa la mitad del espacio geográfico y poblacional de América del Sur, además de que mantenemos fronteras con todos los países del subcontinente, con la excepción de Chile y Ecuador.

El MERCOSUR fue creado con esa perspectiva, por la necesidad de substituir la retórica de la desconfianza por la de la asociación. El MERCOSUR es un proyecto de convergencia 
multidimensional -económica, política, social, cultural- primero de Brasil y de Argentina, al cual se sumaron Uruguay y Paraguay, nuestros vecinos inmediatos, y ahora Venezuela. Es el símbolo mayor del proceso de superación de rivalidades en la Cuenca del Plata, región que ya experimentó su cuota de conflictos y que, en los años 70, parecía estar bajo el riesgo de ser testigo de una incipiente competencia nuclear, situación de ambigüedad finalmente cerrada por el acuerdo nuclear Brasil-Argentina (1991) y la sucesiva creación de la Agencia Brasileño-Argentina de Contabilidad y Control de Materiales Nucleares (ABACC) y el acuerdo tripartito con la OIEA.

El MERCOSUR es, de esta forma, una expresión del deseo de convivencia armónica de nuestros pueblos, de superación de las rivalidades y del desarrollo económico mutuo. No constituye un mero proceso coyuntural o iniciativa de un partido, es un proyecto de Estado, multipartidario. Ajustes se pueden realizar a lo largo del camino, pero la esencia del proyecto del MERCOSUR no es objeto de crítica por parte de ninguna fuerza política o social en Brasil.

La dimensión político-social del MERCOSUR es una razón suficiente para su existencia. No obstante, el MERCOSUR ha sido una herramienta fundamental para el desarrollo económico de sus países miembros. Si la coyuntura puede, en algunos momentos, parecer desfavorable, el análisis histórico demuestra que el bloque puede enorgullecerse de importantes avances. El flujo de comercio intra-Mercosur creció 900\% en los veinte años posteriores a la firma del Tratado de Asunción en 1991, resultado superior, por tanto, a la evolución de nuestro comercio con el resto del mundo. Además, en el caso de Brasil, 90\% de las exportaciones hacia el MERCOSUR son productos industrializados, frente a solo un $5 \%$ de nuestras exportaciones hacia China, por ejemplo. La liberalización arancelaria es próxima al $100 \%$ en el MERCOSUR, que también presenta altas tasas de liberalización con los demás socios comerciales sudamericanos. El cronograma de desgravación prevé que, en 2019, el MERCOSUR tendrá 100\% de su comercio con aranceles cero con Bolivia, 94,4\% con Ecuador, 99,8\% con Perú, y 83,6\% con Colombia.

La relación entre Chile y el MERCOSUR es un caso especial. Se trata de un país asociado al bloque, y nuestro comercio 
se rige por el Acuerdo de Complementación Económica (ACE) 35, firmado en 1997. De acuerdo con el cronograma de desgravación arancelaria, los últimos aranceles entre nuestros países fueron eliminados en enero de 2014. Por esa razón, Brasil es, hoy, el mercado más abierto para los productos chilenos en toda América Latina.

Nuestro mercado no es solo el más abierto, sino que también el de mayor volumen. Brasil -aisladamente- importa más productos chilenos que el conjunto de los miembros de la Alianza del Pacífico. Nuestro mercado se ha mostrado más resiliente también: en el período 2012-2014 las exportaciones chilenas hacia Brasil han presentado estabilidad, frente al retroceso mostrado por las exportaciones chilenas hacia los miembros de la Alianza. También, observamos con satisfacción el hecho de que nuestro comercio bilateral es equilibrado e involucra un número significativo de pequeñas y medianas empresas de los dos países, lo que termina por apalancar a este sector responsable de la mayor parte de la generación de empleos en nuestras economías.

Brasil es el cuarto mayor socio comercial de Chile, después de China, de Estados Unidos y de Japón. En el primer semestre de 2014, el intercambio bilateral creció a un ritmo de 5,1\%, lo que destaca aún más al contrastarlo con la baja de 3,7\% registrada por el intercambio comercial total de Chile con el mundo. De esa forma, la participación de Brasil en el comercio global de Chile pasó de 5,7\% a 6,3\% del total. El resultado es todavía más significativo si tomamos en cuenta que el comercio de Chile con sus socios de la Alianza del Pacífico se contrajo $13,7 \%$, rango similar a la contracción del comercio de Chile con los países del NAFTA, de 12,5\%. Ni aún el intercambio de Chile con Asia - crecimiento de 3\%-presentó un dinamismo equivalente al del intercambio de Chile con Brasil en ese período.

Además de importante socio comercial, Brasil es también el principal destino de las inversiones chilenas en el mundo. La DIRECON recientemente estimó en cerca de US\$ 25.000 millones el stock acumulado de inversiones chilenas en Brasil, involucrando a más de 150 empresas y correspondiendo a casi un cuarto del total invertido por Chile en el exterior. Esas inversiones son el hilo conductor de una integración 
económica profunda, bien reflejada en la fusión de grandes empresas como LAN y TAM, o en la extensa presencia en Brasil de empresas chilenas como CMPC y CENCOSUD. En sentido contrario, diversas empresas brasileñas, como PETROBRÁS, ITAÚ, BTG PACTUAL, GERDAU, entre otras, ya se encuentran firmemente establecidas en Chile. Entendemos que la inversión recíproca deberá crecer aún más en la medida en que nuestras dos economías vayan aumentando el intercambio y el conocimiento mutuo. El gobierno brasileño, junto con la presidenta Bachelet, trabajarán por la integración económica y el aprovechamiento de las sinergias que existen entre nuestras sociedades.

No puedo terminar estas consideraciones sin referirme al tema de la supuesta división entre una América del Sur del «Pacífico» y otra América del Sur del "Atlántico». Es posible llegar al Asia por cualquiera de los dos océanos, inclusive con costos similares. El acelerado avance del comercio con Asia -se da, sobre todo, con China- es un fenómeno que ocurre de manera equivalente en todos nuestros países. El nivel de ese comercio, basado en la exportación de commodities y en la importación de manufacturados, también es idéntico en Perú o Uruguay, Chile o Brasil.

La retórica de esa división necesita ser superada, y es exactamente en ese sentido que se discute la eventual convergencia entre los proyectos del MERCOSUR y de la Alianza del Pacífico. Desearía resaltar el liderazgo chileno en este movimiento de aproximación, lanzado por la presidenta Michelle Bachelet y por el canciller Heraldo Muñoz desde los primeros días de este nuevo período de gobierno. Este proceso fue objeto de atención de nuestros ministros de Relaciones Exteriores en la $1^{\circ}$ Reunión Informativa realizada en la Ciudad de Cartagena, el pasado 1 de noviembre. A su vez, la cancillería chilena organizará, el próximo 24 de noviembre, un seminario sobre el mismo tema, ocasión en que serán discutidas las posibles áreas de convergencia. Cabe registrar la intensa agenda que se formula alrededor de ese proceso, el cual ha obtenido la atención especial de nuestros cancilleres. Entre los diversos temas que han sido citados como áreas de interés común, de manera aún prospectiva, están aquellos relacionados con la libre circulación de personas, la armonización de reglamentos 
y de procesos aduaneros, certificados de origen, entre otros. Este es un proceso que recién se inicia, de manera que otros ítems deberán ser agregados a la lista.

En el plano bilateral observamos con atención una especie de termómetro de la intensidad e importancia de la relación bilateral, que es la frecuencia de visitas mutuas entre nuestras principales autoridades. En el caso de Brasil y Chile, la agenda de visitas ha sido particularmente intensa desde la asunción al mando de Bachelet. Además de las visitas recíprocas de nuestras dos presidentas, observamos que nuestro canciller, el ministro Alberto Figueiredo, visitó Santiago en tres ocasiones distintas. A su vez, Muñoz decidió elegir a Brasil como destino de su primer viaje oficial. Otras visitas ministeriales, como la del ministro de Defensa de Chile a Brasil y del ministro de Pesca de Brasil a Chile, fueron realizadas; diversos mecanismos bilaterales se establecieron o están siendo reactivados, dedicados a las consultas políticas, comercio bilateral, energía, cooperación técnica, ciencia y tecnología.

Brasil y Chile comparten, de esta forma, una diversificada agenda de intereses comunes, que es reflejo también de nuestra historia, de nuestra cultura común ibero-americana y de nuestra geografía. Por tanto, trataremos de promover la convergencia y la integración de nuestras economías y sociedades, en un marco de democracia y respeto a los derechos humanos. 